\title{
POST-MASTECTOMY EXTERNAL BEAM RADIATION FOLLOWED BY HDR SURFACE MOULD BRACHYTHERAPY BOOST VS. EXTERNAL BEAM RADIOTHERAPY ALONE IN HIGH-RISK CASES- A RANDOMISED CONTROLLED TRIAL
}

\author{
Arkoprovo Halder ${ }^{1}$, Partha Sarathi Halder², Anjan Das³, Soumita Podder 4 , Sandip Roy Basunia 5 , Tapobrata Mitra ${ }^{6}$, Hirak Biswas $^{7}$, \\ Subrata Kumar Mandal ${ }^{8}$
}

${ }_{1}^{1}$ Senior Resident, Department of Radiotherapy, R. G. Kar Medical College and Hospital, Kolkata, West Bengal, India. ${ }^{2}$ Associate Professor, Department of Anaesthesiology, College of Medicine and Sagore Dutta Hospital, Kolkata, West Bengal, India. ${ }_{3}^{3}$ Associate Professor, Department of Anaesthesiology, College of Medicine and Sagore Dutta Hospital, Kolkata, West Bengal, India. ${ }_{4}^{4}$ Assistant Professor, Department of Radiotherapy, Murshidabad Medical College and Hospital, Berhampore, West Bengal, India. ${ }^{5}$ Assistant Professor, Department of Anaesthesiology, Midnapore Medical College, West Midnapore, West Bengal, India. ${ }^{6}$ Assistant Professor, Department of Anaesthesiology, Murshidabad Medical College and Hospital, Berhampore, West Bengal, India. ${ }^{7}$ Assistant Professor, Department of Anaesthesiology, College of Medicine and Sagore Dutta Hospital, Kolkata, West Bengal, India. ${ }^{8}$ Professor and HOD, Department of Anaesthesiology, College of Medicine and Sagore Dutta Hospital, Kolkata, West Bengal, India.

\section{ABSTRACT}

\section{BACKGROUND}

Post-mastectomy radiotherapy (phase III) results in excellent local control and better survival in patients with higher risk pathological scenario. Involved or closed surgical margins were not regarded as high risk, but surprisingly, they were susceptible to local failure. Boost dose followed by chest wall radiation, however, is the modality of choice in this hospital setting.

\section{MATERIALS AND METHODS}

Between 2007 and 2012, 60 high-risk post-mastectomy patients were identified and were randomly assigned into study and control arms (30 each). The study arm was given Post-Operative Radiotherapy (PORT) with chest wall dose of 50 Gy/25\# fractions followed by Brachytherapy boost dose of $7.5 \mathrm{~Gy} / 3 \#$. Patients in control arm were treated with PORT alone.

\section{RESULTS}

A continuous follow-up of 36.3 months (median), 7 were alive with recurrent disease ( 1 in study and 6 in control arm) and 3 had died ( 1 in study and 2 in control arm). There was comparable grade 3 skin toxicity between the two arms with no case of late lung toxicity at 12 months in either arms. The 3-year local control (96.7\% vs. 79.2\%, p= 0.044) and Disease-Free Survival (DFS) of 3 years (93.3\% vs. $73.3 \%, \mathrm{p}=0.041)$ were better in study arm, but 3-year overall (OS) survival (96.7\% vs. 93.3\%\%, p= 0.55) was comparable.

\section{CONCLUSION}

Surface-mould HDR (High Dose Rate) brachytherapy boost after EBRT (External Beam Radiation Therapy) is safe and feasible for treating high-risk post-mastectomy cases with improved 3 years local control rates and DFS, but has no 3 years OS benefit.

\section{KEY WORDS}

Brachytherapy; Loco-Regional Recurrence (LRR), Post-Mastectomy Radiation Therapy (PMRT), HDR (High Dose Rate) Surface Mould; Disease-Free Survival (DFS); External Beam Radiotherapy (EBRT), Supraclavicular Lymph Node (SCLN), Clinical Target Volume (CTV), Surgical Scar Boost.

HOW TO CITE THIS ARTICLE: Halder A, Halder PS, Das A, et al. Post-mastectomy external beam radiation followed by HDR surface mould brachytherapy boost vs. external beam radiotherapy alone in high-risk cases- a randomised controlled trial. J. Evolution Med. Dent. Sci. 2018;7(43):4642-4647, DOI: 10.14260/jemds/2018/1036

\section{BACKGROUND}

Trials have been found to demonstrate that post-mastectomy radiation therapy attenuates the probability of loco-regional recurrence by (50-65\%) for women exhibiting high-risk characteristics after mastectomy.[1-4] Tumour size $\geq 5 \mathrm{~cm}$, and/ or 4 or $\geq$ axillary node involvement and invasion of the skin were regarded as the high-risk pathological features.[2-4]

'Financial or Other Competing Interest': None.

Submission 27-08-2018, Peer Review 05-10-2018,

Acceptance 11-10-2018, Published 22-10-2018.

Corresponding Author:

Dr. Partha Sarathi Halder,

37Q, Raja Manindra Road,

1 st Floor, Paikpara, Kolkata-700037,

West Bengal, India.

E-mail: dr_pshalder@yahoo.com

DOI: $10.14260 /$ jemds/2018/1036
When tumour margin was not properly evaluated, patients' skin and/ or deep fascia involvement (subgroup with high preponderance of margin involvement) was shown to reduce loco-regional recurrence significantly at 10 years (40\% vs. 7\%).[3,4] Regimens [40 Gy over 4 weeks (European protocol) with electrons or 37.5 Gy over 3 weeks (Canadian protocol) via tangents] along with nodal irradiation (axillary as well as internal mammary node).[2,4] Involved or close surgical margins were administered no boost, loco-regional recurrence was (5 - 10\%) among irradiated patients.

Historic cohort studies on patients with node negativity who did not receive PMRT had been documented with higher risk of loco-regional recurrence in presence of involved $(<2$ $\mathrm{mm}$ ) or closed margins when compared with negative margins.[5,6] In this scenario, effect of post-mastectomy radiation therapy is not clear. ${ }^{7,8]}$ Documents from breast conservation studies indicate a "boost" to the breast 
carcinoma bed that attenuates loco-regional recurrence when compared with whole-breast irradiation alone. ${ }^{[9,10]}$ The above mentioned beneficial effect was more evident with enhanced doses in the scenario of involved or closed surgical margins.[11] Registry-based data acquisition suggested locoregional recurrence rate appreciably improved with higherdose radiotherapy.[12,13]

Our current study was indented to investigate the role of "boost" in improvement of local control, patterns of failure, toxicity and survival rates among women with involved or closed margins who received post-mastectomy radiotherapy with boost.

Commonest site for loco-regional failure is scar in the chest wall. Surgical scar areas are boosted with 10 Gy in 5 fractions with enfacing electrons whenever there is a positive margin or close one. Few studies done previously to investigate the role of boost after PMRT with close/ positive margins usually employed electrons and thus it has become "standard" for scar boosting in majority of the centres in the world and also in India.[14] However, in eastern India, there is scarcity of linear accelerators (also electron therapy) in vast majority of government as well as private health care sectors. Also it is a ground reality that ours being a developing nation, vast majority of cancer patients are from low-socioeconomic status and seek treatment in Radiotherapy Department of government tertiary care hospitals. However, fortunately HDR brachytherapy facility is available in our institute as well as majority of other government tertiary care hospitals in eastern India. There is extreme paucity of studies investigating role of HDR surface mould brachytherapy scar boost following PMRT in the setting of close or positive margins except one study from India.[15] But it was basically a pilot study and not a randomised trial comparing brachytherapy boost with control arm. So, in this study we have attempted to compare PMRT followed by brachytherapy boost in the study arm with the control arm treated with PMRT alone in close or positive margin cases in a randomised trial

\section{MATERIALS AND METHODS}

This randomised controlled trial was approved by the Institutional Ethical Committee. The patients were randomised using computer generated random number list and group allocation was done using sealed envelopes. These opaque envelopes had a paper slip inside them indicating either study or control group. On the outside patient's registration number was mentioned. When the patient was received in the Radiotherapy Department, the numbered envelope was handed over to a resident doctor (radiation oncologist) who was not taking part in the study. He opened the envelope and prepared the protocol of radiotherapy to be administered in post-mastectomy patients. The senior radiation oncologists who did not take part in the above study helped in identifying recurrence and side effects.

Female patients, free of cardio or pulmonary comorbidities with unilateral breast cancer and had to undergo mastectomy with positive or closed surgical margins $(\leq 2$ $\mathrm{mm}$ ) were included in this study. 60 patients who fulfilled the inclusion criteria were randomised and distributed into study and control groups ( 30 in each arm) and planned for postmastectomy radiotherapy with sequential scar boost with use of high dose rate flap surface mould. All patients must have undergone MRM before with proper axillary dissection (Minimum of 10 lymph nodes sampled). They must have received standard adjuvant or neo-adjuvant chemotherapy. Sample size was taken as per our convenience.

\section{The Patients were treated as Follows}

Study arm consisted of first received External Beam Radiotherapy (EBRT)/ Post-Mastectomy Radiotherapy (PMRT) to chest wall and SCLN + axilla (if applicable) as 50 Gy (5000 cGy) in 25 conventional fractions (2 Gy single \#/day and 5 days/ week from Monday - Friday). It was followed by sequential HDR-Brachytherapy scar boost using surface mould with dose of 7.5 Gy in 3 fractions, 2 weeks after completion of EBRT (1\# was given daily for 3 successive days).

Control arm consisting of patients was treated with PMRT alone with similar dose prescription.

Acquired data were gathered and analysed on the basis of demographics, tumour characters (Location, grade, histology, hormone receptor status, HER2 status, pathological stage, surgical margin status) and treatment modalities (Radiotherapy as well as systemic therapy).

Chemotherapy prior to operation was not an exclusion factor with both pre- and post-chemotherapy staging related documentations preserved.

\section{Procedure of Delivering EBRT}

The patient was immobilised with thermoplastic immobilisation device with ipsilateral arm abducted (90 degrees) and externally rotated. A large bore CT simulator was used to accommodate the patient in arm position. Wires with radio-opacity character were placed just upon the surgical scar and treatment field. The acquired CT images were converted to DICOM (Digital Imaging and Communication in Medicine) and transferred to TPS (Treatment Planning System). After loading of images and segmentation, areas of disease interest are contoured on CT slices as per radiation therapy oncology guideline contouring guidelines.

\section{Procedure of Brachytherapy}

For chest wall scar boost, CT-image based high dose rate surface mould brachytherapy was given sequential to EBRT. Clinical target volume for post-mastectomy scar boost was not guided by any standard guidelines. In our study, clinical target volume at $5 \mathrm{~mm}$ beneath the skin (CTV_hdr) was a uniform $5 \mathrm{~mm}$ thick volume drawn and planned boost area was marked with radio-opaque wires, so that it can be visible on CT images. 7.5 Gy in 3 fractions, that means 2.5 Gy in every fraction was the prescribed dose for CTV_hdr.

Commercially available CT compatible 6 flexible channels were used in our study for high dose rate surface mould therapy. The mould is constructed upon a thermoplastic immobilisation cast with wax mould that holds the channels in position securely; $1 \mathrm{~cm}$ gap was kept between every two channels and this flap was capable of treating the desired area of interest. Slightly radio-opaque catheters were used and thus were visible on the CT images. Eclipse Brachyvision $^{\mathrm{TM}}$ (Version 10.0.42) Varian Medical Systems, Inc., Hansen Way, Palo Alto, CA, was used for optimisation and calculations. 
Thermoplastic cast, made of full carbon fibre breast board, was used for immobilisation of the patients in the supine posture with the same arm abducted above head for easy image registration on EBRT. Oncologists made surface markings at a gap of $3 \mathrm{~cm}$ in craniocaudal axis and mediolateral borders are kept at a distance of $2-3 \mathrm{~cm}$ away from the scar. External beam radiotherapy casts were prepared separately. Catheter flap was kept in the cast for chest wall scar boost, so that it remained on patient's chest wall and its same position was ensured throughout the entire treatment associated with subsequent fractions. In external beam radiotherapy and high dose rate treatment, the identical position of the patients were maintained for easy image registrations for planning sums to be generated later for evaluation [Figure 1].

\section{Dose Prescription}

External beam radiotherapy dose in our study was 50 Gy in 25 fractions to the entire affected side chest wall and regional nodes given to all patients to both study and control arm. CTV_hdr dose was kept at 2.5 Gy in every fraction, delivered over 3 fractions. Thus, in study arm, total target scar boost dose was 7.5 Gy for all the patients. High dose rate surface mould dosing in our study was kept much less than ACR guidelines. We used 7.5 Gy in 3 fractions, whereas ACR guidelines recommended 10 Gy in 5 fractions. Thus, administering 10 Gy in 5 fractions with surface mould can cause more skin reactions. Brachytherapy is started 2 weeks after completion of EBRT. Excellent plans for proper evaluation are the ones whose V100\% is at least 95\%. Dose distributions are meticulously evaluated slice by slice, qualitatively and then quantitatively by the use of Dose Volume Histograms (DVHs).

\section{Evaluation during Treatment and Follow-Up}

During treatment acute toxicities like skin, haematological, cardio-respiratory problems in every patient were evaluated carefully weekly. After completion of the radiation protocol, all patients were followed up monthly for 1 st 6 months, then 2 monthly upto 1 year and then 3 monthly for 2 nd and 3 rd year.

Skin toxicity evaluation was done weekly during radiation for acute toxicity and done 1 monthly after radiation and during follow-up for delayed toxicity. For pulmonary toxicity assessment, all were subjected to pulmonary function tests (Clinical, CT Chest, FVC) done at baseline (prior to radiotherapy) and at the end of radiation ( 6 weeks after) and during subsequent follow-up (6 months and 12 months). Assessment of respiratory distress, cough and chest pain will be done at each visit. CECT thorax will be done if patient is symptomatic or there are significant alterations in PFT from normal.

Loco-regional control, disease-free survival, toxicities and overall survival were the major outcome variables which were measured in our study. From the date of initial tissue diagnosis (biopsy) to first detection of an event was taken as the time to reach the endpoint.

\section{Statistical Analysis}

Acute and late toxicities will be assessed using the Standard Acute Radiation Morbidity Scoring Criteria and Late Radiation Morbidity Scoring Scheme and compared using
Chi-square test. SPSS 21.0 (SPSS, Inc., Chicago, IL) was used for analysing demographic and clinical characteristics of patients. Disease control and survival was described using Kaplan-Meier method.

\section{RESULTS}

From 2007 to 2012, 60 high-risk post-mastectomy patients were identified and were randomly assigned into study and control arms (30 each). [Table 1] shows the demographic profile of the patients. All of our patients had no history of cancer and were females. All patients were suffering from invasive carcinoma as well as lymphovascular involvement and were specifically identified in 41 patients ( 22 in study arm and 19 in control arm). There was no any case of in situ Ductal carcinoma or Lobular carcinoma.

23 patients underwent neoadjuvant chemotherapy for 6 cycles before mastectomy (10 in study arm and 13 in control arm); 37 patients who did not receive chemotherapy underwent mastectomy within 2 weeks (2 - 39 days) of biopsy confirmation. All the patients along with mastectomy had complete axillary lymph node staging and the number of lymph nodes removed was 13 (10 - 24). For 46 patients with involved lymph nodes (25 in study arm and 21 in control arm), the number of involved nodes were $5(2-21)$ with extra-nodal extension observed in 22 patients $(9$ and 13 in study and control arm respectively).

29 patients (16 and 13 in study and control arm respectively) had undergone additional radiotherapy to the regional lymphatics over chest wall radiation with 5000 cGy. All the patients were followed up for a median duration of 36.3 months. In study group alive patients without recurrence were 28, alive but recurrent disease in 1 patient (loco-regional recurrence at axilla at 16 months) and death in 1 patient (without any recurrence). But in control group alive patients without recurrence were 22 , alive but recurrent disease in 6 patients $(3$ suffered from loco-regional recurrence at chest wall and 3 from nodal recurrence with median survival of 20 months) and death in 2 patients (without any recurrence).

Grade III acute skin toxicities were comparable among two groups. There was no acute grade III or IV pulmonary and haematological toxicity in either arm. No case of persistent late lung toxicity at 12 months in either arm.

As demonstrated in [Figure 2] and [Figure 3], the 3-year local control ( $96.7 \%$ vs. $79.2 \%, \mathrm{p}=0.044)$ and 3-year diseasefree survival ( $93.3 \%$ vs. $73.3 \%, \mathrm{p}=0.041$ ) respectively were better in study arm, but from [Figure 4] the 3-year overall survival $(96.7 \%$ vs. $93.3 \%, \mathrm{p}=0.55)$ was comparable in both arms.

\begin{tabular}{|c|c|c|c|}
\hline Variable & $\begin{array}{c}\text { Study Arm } \\
(\mathbf{N = 3 0 )}\end{array}$ & $\begin{array}{c}\text { Control Arm } \\
\text { (N= 30) }\end{array}$ & P value \\
\hline $\begin{array}{c}\text { Age at Diagnosis } \\
\text { (yrs.) }\end{array}$ & $55.2 \pm 7.2$ & $59 \pm 10.2$ & 0.0705 \\
\hline $\begin{array}{c}\text { Histology } \\
\text { Invasive ductal } \\
\text { Invasive lobular } \\
\text { Others }\end{array}$ & $\begin{array}{c}24(80 \%) \\
5(16.6 \%) \\
1(3.3 \%)\end{array}$ & $\begin{array}{c}27(90 \%) \\
3(10 \%) \\
0(0 \%)\end{array}$ & 0.3370 \\
\hline $\begin{array}{c}\text { Laterality } \\
\text { Left/ Right }\end{array}$ & $\begin{array}{c}19(63.3 \%) / 11 \\
(36.6 \%)\end{array}$ & $\begin{array}{c}15 \\
(50 \%) / 15(50 \%)\end{array}$ & 0.1296 \\
\hline $\begin{array}{c}\text { Oestrogen } \\
\text { Receptor }\end{array}$ & $24(80 \%)$ & $21(70 \%)$ & 0.1709 \\
\hline
\end{tabular}




\begin{tabular}{|c|c|c|c|}
\hline $\begin{array}{c}\text { Positive } \\
\text { Negative }\end{array}$ & $6(20 \%)$ & $9(30 \%)$ & \\
\hline $\begin{array}{c}\text { Progesterone } \\
\text { Receptor } \\
\text { Positive }\end{array}$ & $17(56.6 \%)$ & $20(66.6 \%)$ & 0.2690 \\
Negative & $13(43.3 \%)$ & $10(33.3 \%)$ & \\
\hline $\begin{array}{c}\text { Her-2/neu } \\
\text { Status }\end{array}$ & & & \\
Positive & $8(26.7 \%)$ & $5(16.7 \%)$ & 0.2154 \\
Negative & $22(73.3 \%)$ & $25(83.3 \%)$ & \\
\hline Surgical Margin & & & \\
Status & $16(53.3 \%)$ & $18(60 \%)$ & 0.2114 \\
Positive/Involved & $7(23.3 \%)$ & $9(30 \%)$ & \\
$<1$ mm & $7(23.3 \%)$ & $3(10 \%)$ & \\
1-2 mm & & & \\
\hline Table 1. Demographic Characteristics and Risk Factors \\
\hline
\end{tabular}

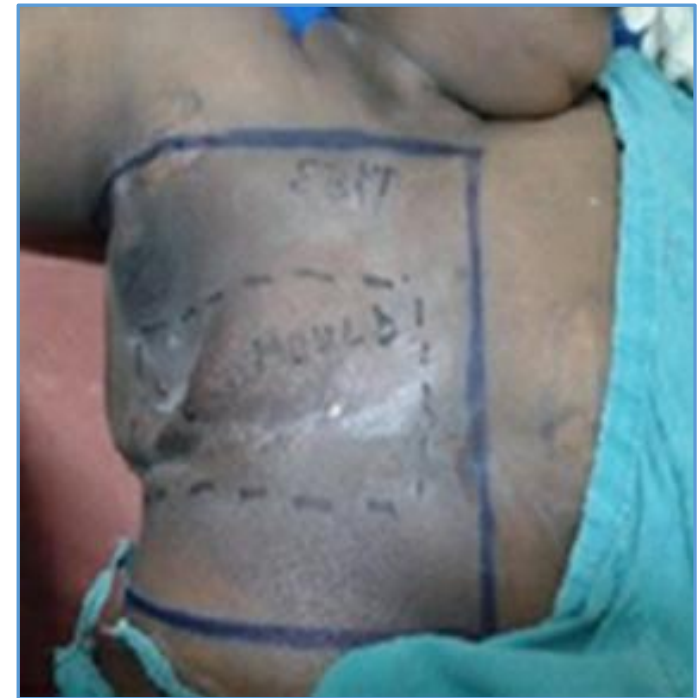

Figure 1 (a). EBRT Field and Smaller Field covered by Surface Mould Brachytherapy

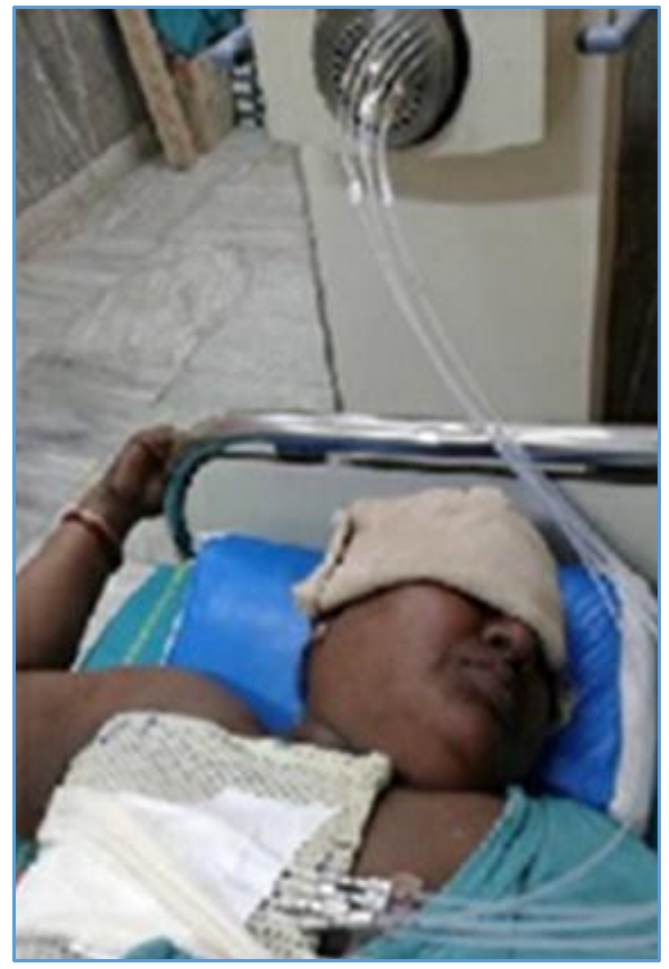

Figure 1 (b). Surface Mould Brachytherapy Flap with 6 Channels

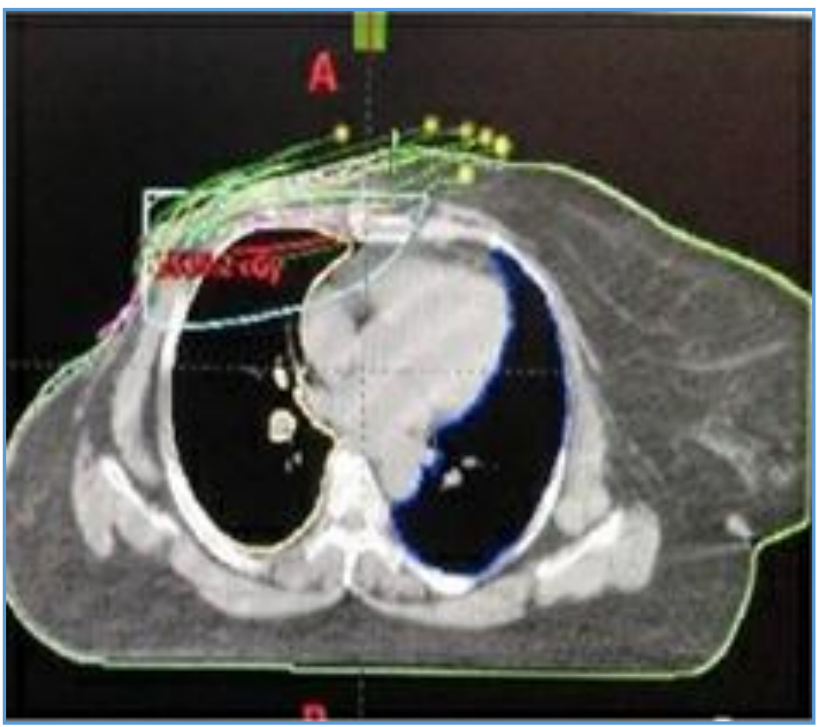

Figure 1 (c). Dose distribution of CTV HDR

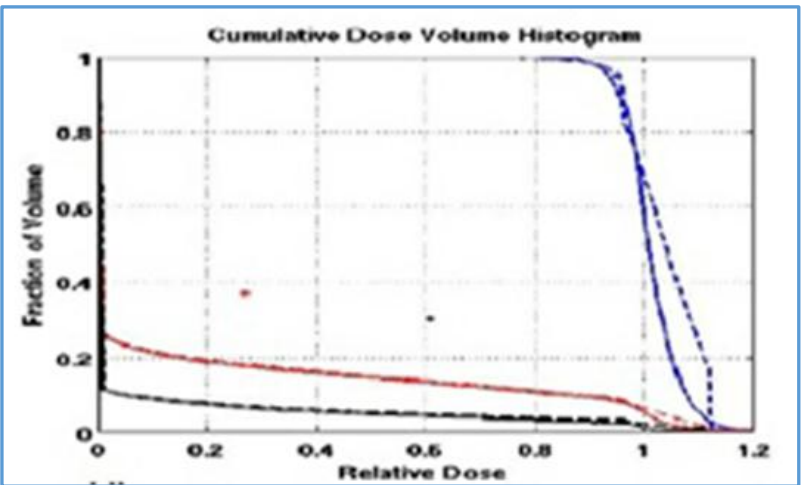

Figure 1 (d). Cumulative DVH

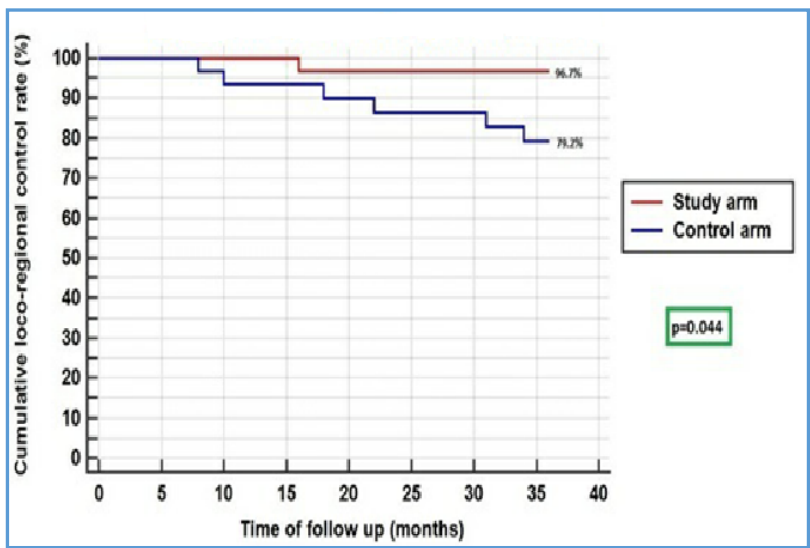

Figure 2. Three-Year Loco-Regional Control Rates

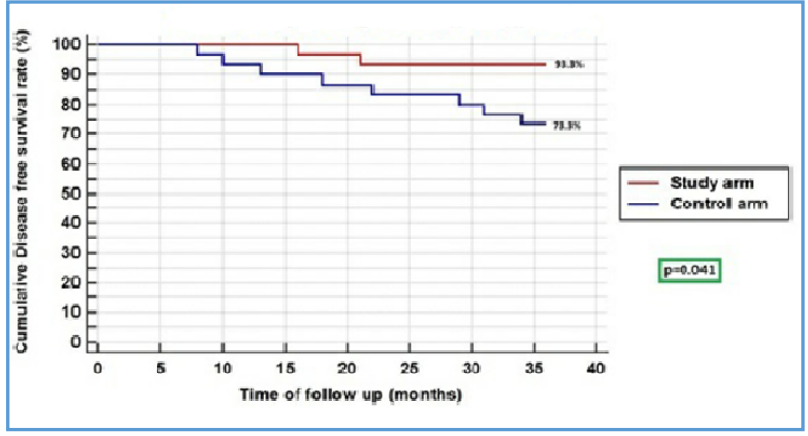

Figure 3. Three-Year Disease-Free Survival Rates 


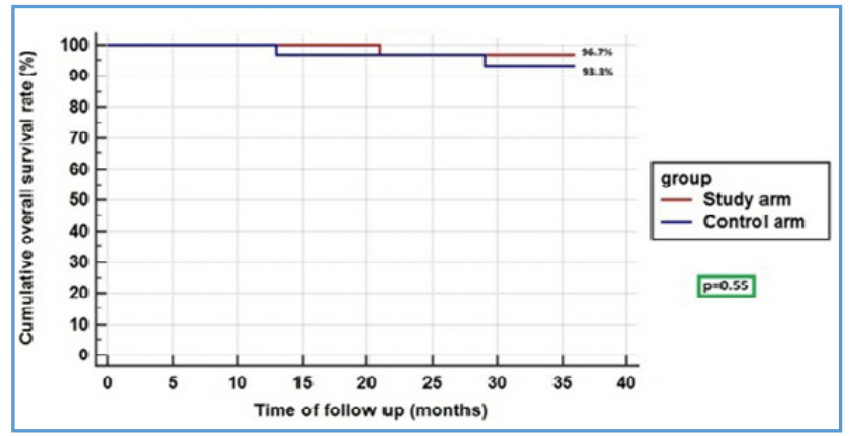

Figure 4. Three-Year Overall Survival Rates

\section{DISCUSSION}

Following mastectomy, loco-regional recurrence is an unpredictable and worrisome site for carcinoma recurrence. Previous data suggests that a vast majority of patients who experience isolated chest wall failure will later develop distant metastasis within a course of 5 years of loco-regional recurrence. Patients with $\mathrm{T}_{1-2} \mathrm{~N}_{0}$ disease has a $25 \%$ enhanced risk of recurrence after mastectomy.[16] In this way, proper local control after mastectomy is very crucial. Phase III randomised trials in Denmark ${ }^{[2,3]}$ and Canada ${ }^{[4]}$ recommend post-mastectomy radiotherapy when tumour size $>5 \mathrm{~cm}$, involvement of pectoralis and/ or skin, inflammatory breast malignancy and/ or lymph node involvement. LRR benefit and enhanced overall survival strengthens the use of postmastectomy radiotherapy. However, surgical margin status was not reported and thus the benefit in this setting has not yet been evaluated prospectively.

In presence of associated high-risk features, loco-regional recurrence chances are higher with close or involved surgical margins.[5-8]

Jagsi et al in a case series observed 64 closed $(<2 \mathrm{~mm})$ or involved margin patients (non-irradiated, node-negative) suffered from 21\% loco-regional recurrences (10 yrs. interval) after mastectomy; whereas another group consisting of 662 patients with margins $>2 \mathrm{~mm}$ suffered from $5 \%$ recurrence with $\mathrm{p}<0.001$. $^{[5]}$ At Fox Chase Cancer Centre, investigators found similar higher 8 yrs. loco-regional recurrences $(24 \%$ vs. $7 \%$ ) in presence of 1 - 3 lymph node involvement.[6] In this way, loco-regional recurrences are closely associated with margin proximity $(<2 \mathrm{~mm})$ which helps us for optimising the dose of post-mastectomy radiotherapy. Feigenberg et al[12] with help of retrospective evidences came to a conclusion that post-mastectomy radiotherapy and boost doses benefit a lot for high-risk patients.

With respect to PMRT and boost doses, a retrospective evidence suggests LRR benefit for general populations of described detailed outcomes for PMRT in a general population of 323 patients treated with electrons to the chest wall.[12] Investigators from University of Miami performed a retrospective study on 582 patients for evaluating relationship between total PMRT dose and loco-regional recurrences.[13] They administered total radiotherapy dose $</>5040$ cGy (standard chest wall doses are 4500 - 5040 cGy). After a median 45 months follow-up, 5 yrs. locoregional recurrences was $5.7 \%$ vs. $12.7 \%$ in patients who completed or did not complete the total dose > 5040 cGy.
Breast cancer with high-risk group like stage (inflammatory) III-IVC disease, triple negative receptor (HER2-negative, hormone-insensitive) disease had a superior outcome with scar boost.

In our study, we too found lower rate of loco-regional recurrence with use of RT boost in closed/ involved margin population with association of established high-risk features. Multiple-quadrant involvement, high-grade ductal carcinoma in situ, closed/ involved margins and/ or younger age like factors may increase this risk.[17-19]

A single arm retrospective study by Johnson et al[20] demonstrated favourable local control (96.9\%) with postmastectomy radiotherapy plus boost with the background of closed/ involved margins after modified radical mastectomy. The modality used for boost was electron beam therapy.[20] However, there are hardly any studies that are comparing role of boost with a control group not receiving boost.

Also, another method for post-mastectomy scar boost is HDR surface mould brachytherapy. It was first described by Vijayaprabhu et al in a single arm pilot study.[15] This protocol is not complicated with use of electron arc and electron beam techniques. Chest being a curve structure and due to presence of lung, bone and soft tissue heterogeneities, a great challenge is being faced for planning of irradiation. Custom made cutouts are being used to collimate the electron beam to the planned area of irradiation.

These electron arcs are still preferred over external beam radiotherapy photons, as these arcs have favourable depth dose characteristics and much better susceptible organ sparing activity. A simple immobilisation cast for ensuring reproducibility over fractions is required in HDR surface mould technique. This cast minimises air gaps between the flap and skin. Sometimes, a planner is used to adjust the dose distribution when optimisation is not satisfactory.

If surgical scar boost is planned as post-mastectomy radiotherapy, then HDR surface mould is definitely a promising technique and should be made a routine choice in clinics.

In our study, we compared brachytherapy scar boost following PMRT vs. PMRT alone in a prospective randomised controlled trial. There were no significant differences in acute haematological, skin or pulmonary toxicities. No late grade III or IV pulmonary or dermatological toxicity was seen at 12 months follow-up. After EBRT, surface mould high dose rate brachytherapy boost is thus relatively safe and practical approach in treating high-risk post-mastectomy patients with improved 3-year local control rates and disease free survival, but no 3-year overall survival benefit. For ensuring this benefit, additional follow-up will be important.

\section{CONCLUSION}

It can be concluded from our study that there is an improved 3-year local regional control rate and 3-year disease free survival achieved with acceptable toxicities in PMRT plus HDR surface mould Brachytherapy boost when compared to PMRT alone in the setting of 'involved or closed margins' after modified radical mastectomy. Longer follow-up with larger sample size is warranted to see if an improved LRC rate translates to long-term improvement in overall survival rates. 


\section{ANNEXURE}

- EBRT- External Beam Radiotherapy.

- HDR- High Dose Rate.

- PMRT- Post-Mastectomy Radiotherapy.

- $\quad$ LRR- Loco-Regional Recurrence.

- $\quad$ Gy-Gray (S.I. unit of Absorbed Dose).

- $\quad$ SCLN- Supraclavicular Lymph Nodes.

- CT-Computerised Tomography.

- HER2- Human Epidermal Growth Factor Receptor 2.

- $\quad$ RTOG- Radiation Therapy Oncology Group.

- CTV-Clinical Target Volume.

- ACR-American College of Radiology.

- DVH- Dose Volume Histogram.

- PFT- Pulmonary Function Test.

- $\quad$ FVC- Forced Vital Capacity.

- $\quad$ LRC- Local Regional Control.

- DFS- Disease Free Survival.

- OS- Overall survival.

- $\quad$ SPSS- Statistical Package for the Social Sciences.

- DCIS- Ductal Carcinoma In Situ.

- LCIS- Lobular Carcinoma In Situ.

- OAR- Organs at Risk.

\section{REFERENCES}

[1] Zellars R. Post-mastectomy radiotherapy. Clin Adv Hematol Oncol 2009;7(8):533-43.

[2] Overgaard M, Hansen PS, Overgaard J, et al. Postoperative radiotherapy in high-risk premenopausal women with breast cancer who receive adjuvant chemotherapy. Danish Breast Cancer Cooperative Group 82b Trial. N Engl J Med 1997;337(14):949-55.

[3] Overgaard $M$, Jensen $M B$, Overgaard $J$, et al. Postoperative radiotherapy in high-risk postmenopausal breast-cancer patients given adjuvant Tamoxifen: Danish breast cancer cooperative group 82c randomised trial. Lancet 1999;353(9165):1641-8.

[4] Ragaz J, Jackson SM, Le N, et al. Adjuvant radiotherapy and chemotherapy in node-positive premenopausal women with breast cancer. $\mathrm{N}$ Engl J Med 1997;337(14):956-62.

[5] Jagsi R, Raad RA, Goldberg S, et al. Locoregional recurrence rates and prognostic factors for failure in node-negative patients treated with mastectomy: implications for postmastectomy radiation. Int J Radiat Oncol Biol Phys 2005;62(4):1035-9.

[6] Freedman GM, Fowble BL, Hanlon AL, et al. A close or positive margin is not an indication for chest wall irradiation except in women aged fifty or younger. Int J Radiat Oncol Biol Phys 1998;41(3):599-605.

[7] Truong PT, Olivotto IA, Speers $\mathrm{CH}$, et al. A positive margin is not always an indication for radiotherapy after mastectomy in early breast cancer. Int J Radiat Oncol Biol Phys 2004;58(3):797-804.
[8] Truong PT, Lesperance M, Culhaci A, et al. Patient subsets with T1-T2, node-negative breast cancer at high locoregional recurrence risk after mastectomy. Int J Radiat Oncol Biol Phys 2005;62(1):175-82.

[9] Bartelink H, Horiot JC, Poortmans P, et al. Recurrence rates after treatment of breast cancer with standard radiotherapy with or without additional radiation. $\mathrm{N}$ Engl J Med 2001;345(19):1378-87.

[10] Bartelink H, Horiot JC, Poortmans PM, et al. Impact of higher radiation dose on local control and survival in breast-conserving therapy of early breast cancer: 10year results of the randomized boost versus no boost EORTC 22881-10882 Trial. J Clin Oncol 2007;25(22):3259-65.

[11] Romestaing P, Lehingue Y, Carrie C, et al. Role of a 10Gy boost in the conservative treatment of early breast cancer: results of a randomized clinical trial in Lyon, France. J Clin Oncol 1997;15(3):963-8.

[12] Feigenberg SJ, Mendenhall NP, Benda RK, et al. Postmastectomy radiotherapy: patterns of recurrence and long-term disease control using electrons. Int J Radiat Oncol Biol Phys 2003;56(3):716-25.

[13] Panoff JE, Takita C, Hurley J, et al. Higher chest wall dose results in improved locoregional outcome in patients receiving postmastectomy radiation. Int J Radiat Oncol Biol Phys 2012;82(3):1192-9.

[14] Jagsi R. Post-mastectomy radiation therapy: an overview for the practicing surgeon. Article ID 212979, ISRN Surg 2013;2013: p. 16.

[15] Vijayaprabhu N, Gunaseelan K, Vivekanandan N, et al. Post-mastectomy scar boost irradiation using HDR surface mould brachytherapy by 3D image-based volume optimization. International Journal of Medical Physics, Clinical Engineering and Radiation Oncology 2013;2(4):139-46.

[16] Chagpar A, Kuerer HM, Hunt KK, et al. Outcome of treatment for breast cancer patients with chest wall recurrence according to initial stage: implications for post-mastectomy radiation therapy. Int J Radiat Oncol Biol Phys 2003;57(1):128-35.

[17] Rashtian A, Iganej S, Amy Liu IL, et al. Close or positive margins after mastectomy for DCIS: pattern of relapse and potential indications for radiotherapy. Int J Radiat Oncol Biol Phys 2008;72(4):1016-20.

[18] Eulau SM, Beatty JD. The role of adjuvant radiotherapy after mastectomy in ductal carcinoma in situ, breast. Int J Radiat Oncol Biol Phys 2007;69(3):S228.

[19] Chan LW, Rabban J, Hwang ES, et al. Is radiation indicated in patients with ductal carcinoma in situ and close or positive mastectomy margins? Int J Radiat Oncol Biol Phys 2011;80(1):25-30.

[20] Johnson L, Lichter N, Hextall M, et al. Mastectomy scar boost results in low risk of locoregional recurrence in the setting of close or involved surgical margins. Journal of Cancer Therapy 2014;5:167-71. 